\title{
Redshift drift constraints on $f(T)$ gravity
}

\author{
Jia-Jia Geng, ${ }^{1}$ Rui-Yun Guo, ${ }^{1}$ Dong-Ze He, ${ }^{1}$ Jing-Fei Zhang, ${ }^{1}$ and Xin Zhang*1, ${ }^{2, \dagger}$ \\ ${ }^{1}$ Department of Physics, College of Sciences, Northeastern University, Shenyang 110004, China \\ ${ }^{2}$ Center for High Energy Physics, Peking University, Beijing 100080, China
}

We explore the impact of the Sandage-Loeb (SL) test on the precision of cosmological constraints for $f(T)$ gravity theories. The SL test is an important supplement to current cosmological observations because it measures the redshift drift in the Lyman- $\alpha$ forest in the spectra of distant quasars, covering the "redshift desert" of $2 \lesssim z \lesssim 5$. To avoid data inconsistency, we use the best-fit models based on current combined observational data as fiducial models to simulate 30 mock SL test data. We quantify the impact of these SL test data on parameter estimation for $f(T)$ gravity theories. Two typical $f(T)$ models are considered, the power-law model $f(T)_{P L}$ and the exponential-form model $f(T)_{E X P}$. The results show that the SL test can effectively break the existing strong degeneracy between the present-day matter density $\Omega_{m}$ and the Hubble constant $H_{0}$ in other cosmological observations. For the considered $f(T)$ models, a 30-year observation of the SL test can improve the constraint precision of $\Omega_{m}$ and $H_{0}$ enormously but cannot effectively improve the constraint precision of the model parameters.

PACS numbers: 95.36.+x, 98.80.Es, 98.80.-k

Keywords: redshift drift, cosmological constraints, dark energy, modified gravity, $f(T)$ gravity

\section{INTRODUCTION}

Redshift drift observation directly measures the expansion rate of the universe in the "redshift desert" of $2 \lesssim z \lesssim 5$, which is not covered by existing cosmological observations. Therefore, it could be an important supplement to the other cosmological observations. In 1962, Sandage first proposed to directly measure the variation of the redshift of distant sources [1]. Then in 1998, Loeb suggested the possibility of detecting redshift drift by decades-long observation of the Lyman- $\alpha$ forest in the spectra of distant quasars (quasi-stellar objects, QSOs) [2]. Thus, redshift drift measurement is also referred to as the Sandage-Loeb (SL) test. The 39-m European Extremely Large Telescope (E-ELT) (under construction) is equipped with a high-resolution spectrograph called CODEX (COsmic Dynamics EXperiment), which is designed to collect such SL test signals. A great amount of work has been done on the effect of the SL test on cosmological parameter estimation [3-9]. As far as we know, in most existing works, the best-fit $\Lambda$ cold dark matter model is usually chosen as the fiducial model in simulating the mock SL test data. When these simulated SL test data are combined with other actual data, tension may exist inside the combined data.

In our previous works [10-12], we quantified the impact of future redshift drift measurement on parameter estimation for different dark energy models. This work was based on 30 QSOs, because only about 30 QSOs will be bright enough or lie at a high enough redshift to allow observation of the redshift drift using a telescope such as the E-ELT, according to a Monto Carlo simulation [13].

\footnotetext{
*Corresponding author

$\dagger$ Electronic address: zhangxin@mail.neu.edu.cn
}

To simulate mock SL test data that are consistent with other actual observations, we choose the best-fit dark energy models as the fiducial models in the fit to current data. We find that the SL test data alone cannot tightly constrain dark energy models because of the lack of lowredshift data. However, when combined with other actual observations, the SL test can effectively break the existing parameter degeneracies in current observations and greatly improve the precision of parameter estimation for widely studied dark energy models [10-12].

It is well known that aside from the theory of dark energy [14-38], other explanations for cosmic acceleration exists; one of the most popular is a modification of Einstein's general relativity, i.e., modified gravity (MG) [3956]. Hence, we are very curious about the possible impact of the SL test on cosmological constraints for $\mathrm{MG}$ theories. If the SL test can also effectively improve the constraint results of MG models, we may further affirm the conclusion in Ref. [12] that the improvement of parameter estimation by SL test data is independent of the cosmological models in the background. In this paper, we focus on one popular type of MG theory, $f(T)$ theories. In $f(T)$ gravity, the torsion scalar $T$ in the Lagrangian density is replaced by a generalized function $f(T)$, thus producing a possible mechanism for the cosmic acceleration. We take two typical models as examples, the powerlaw model $f(T)_{P L}[46]$ and the exponential-form model $f(T)_{E X P}$ [47], and quantify the impact of redshift drift measurement on parameter estimation for these models.

We adopt the $f(T)_{P L}$ model with the form

$$
f(T)=\alpha(-T)^{n} .
$$

Here $n$ is the model parameter, and

$$
\alpha=\left(6 H_{0}^{2}\right)^{1-n} \frac{1-\Omega_{m}-\Omega_{r}}{2 n-1},
$$

where $H_{0}$ is the Hubble constant. $\Omega_{m}$ and $\Omega_{r}$ are the 
present-day density parameters for the matter and radiation, respectively.

We adopt the $f(T)_{E X P}$ model with the form

$$
f(T)=m T_{0}\left(1-e^{-p \sqrt{T / T_{0}}}\right) .
$$

Here $p$ is the model parameter, $T_{0}=-6 H_{0}^{2}$, and

$$
m=\frac{1-\Omega_{m}-\Omega_{r}}{1-(1+p) e^{-p}} .
$$

\section{METHODOLOGY}

We first constrain the $f(T)$ models using a combination of current data and then choose the best-fit models as fiducial models in producing 30 mock SL test data. Finally, we constrain the $f(T)$ models again using the simulated SL test data combined with the current data and quantify the improvement in the parameter estimation.

In our analysis, the most typical and commonly used current observations are chosen, i.e., the type Ia supernovae $(\mathrm{SNe})$, the cosmic microwave background (CMB), the baryon acoustic oscillation (BAO), and direct measurement of the Hubble constant $H_{0}$. We use the SNLS3 compilation with a sample of $472 \mathrm{SNe}$ [57] for the SN data. We use the BAO data presented in Ref. [58], i.e., the $r_{s} / D_{V}(z)$ measurements from the 6dFGS [59], SDSSDR7 [60], SDSS-DR9 [61], and WiggleZ [62] surveys. Because we focus on the geometric measurements in this work, for the CMB data, we use the Planck distance priors in Ref. [63]. We use the direct measurement of $H_{0}$ from the Hubble Space Telescope, $H_{0}=73.8 \pm 2.4 \mathrm{~km}$ $\mathrm{s}^{-1} \mathrm{Mpc}^{-1}$ [64].

In the redshift drift measurement, the redshift variation is defined as the spectroscopic velocity shift [2]

$$
\Delta v \equiv \frac{\Delta z}{1+z}=H_{0} \Delta t_{o}\left[1-\frac{E(z)}{1+z}\right]
$$

where $\Delta t_{o}$ is the time interval of the observation. $E(z)=$ $H(z) / H_{0}$ is determined by specific $f(T)$ models; for details, see Ref. [54].

According to a Monte Carlo simulation in Ref. [13], the uncertainty of $\Delta v$ can be expressed as

$\sigma_{\Delta v}=1.35\left(\frac{S / N}{2370}\right)^{-1}\left(\frac{N_{\mathrm{QSO}}}{30}\right)^{-1 / 2}\left(\frac{1+z_{\mathrm{QSO}}}{5}\right)^{x} \mathrm{~cm} \mathrm{~s}^{-1}$,

where $S / N$ is the signal-to-noise ratio defined per 0.0125 $\AA$ pixel, and the last exponent is $x=-1.7$ for $2<z<4$ and $x=-0.9$ for $z>4$. $N_{\mathrm{QSO}}$ is the number of observed QSOs, and $z_{\mathrm{QSO}}$ denotes their redshift. We simulate $N_{\mathrm{QSO}}=30$ SL test data uniformly distributed over six redshift bins of $z_{\mathrm{QSO}} \in[2,5]$. We calculate the central values of the mock data by substituting the obtained best-fit parameters in the fit to the current data into Eq. (5). The error bars can be computed from Eq. (6).
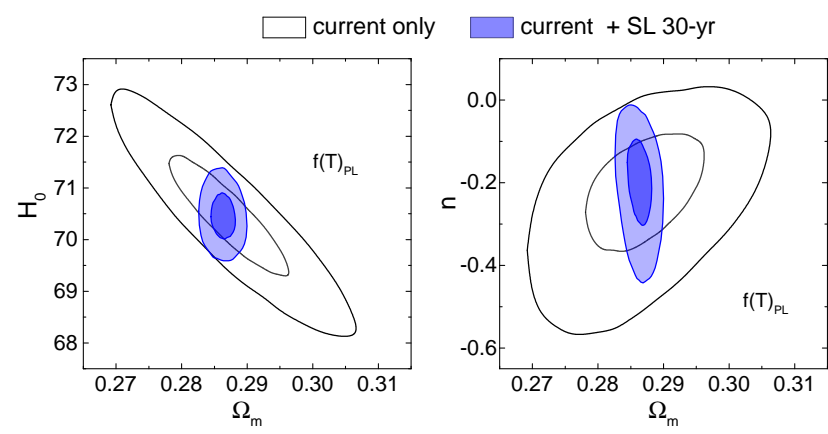

FIG. 1: Constraints (68.3\% and $95.4 \%$ CL) in the $\Omega_{m}-H_{0}$ plane and in the $\Omega_{m}-n$ plane for $f(T)_{P L}$ model with current only and current+SL 30-year data.
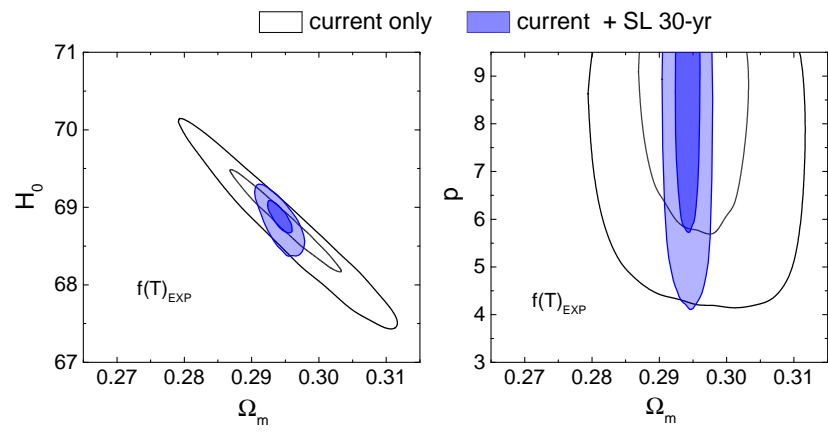

FIG. 2: Constraints $\left(68.3 \%\right.$ and $95.4 \%$ CL) in the $\Omega_{m}-H_{0}$ plane and in the $\Omega_{m}-p$ plane for $f(T)_{E X P}$ model with current only and current+SL 30-year data.

\section{RESULTS AND DISCUSSION}

Table I gives the detailed fit results for the $f(T)_{P L}$ and $f(T)_{E X P}$ models using only the current data (currentonly) and the current data plus the 30-year SL data (current+SL 30-year). We quote $\pm 1 \sigma$ errors, but as the parameter $p$ cannot be well constrained, we quote the $95.4 \%$ confidence level (CL) lower limits. The joint constraints on the $f(T)_{P L}$ model in the $\Omega_{m}-H_{0}$ and $\Omega_{m}-n$ planes are shown in Figure 1. The $68.3 \%$ and $95.4 \%$ CL posterior distribution contours are shown, where the current-only and the current+SL 30-year results are shown in white and blue, respectively. For the current-only data, the precisions of $\Omega_{m}, H_{0}$, and $n$ are constrained to the $4.70 \%$, $2.40 \%$, and $108.97 \%$ level, respectively, whereas for the current+SL 30-year data, the precisions of $\Omega_{m}, H_{0}$, and $n$ are constrained to the $0.97 \%, 0.93 \%$, and $81.29 \%$ level, respectively. Using the SL 30-year combined data can clearly improve the precisions of $\Omega_{m}$ and $H_{0}$ significantly but can improve the constraint precision of parameter $n$ only moderately.

In Figure 2, we present the joint constraints on the $f(T)_{E X P}$ model $\left(68.3 \%\right.$ and $95.4 \% \mathrm{CL}$ ) in the $\Omega_{m}-H_{0}$ 
TABLE I: Fit results for the $f(T)_{P L}$ and $f(T)_{E X P}$ models using the current only and current+SL 30-year data. We quote $\pm 1 \sigma$ errors, but for the parameters that cannot be well constrained, we quote the $95.4 \%$ CL lower limits.

\begin{tabular}{cccccc}
\hline \hline & \multicolumn{2}{c}{ current only } & & \multicolumn{2}{c}{ current + SL 30-year } \\
\cline { 2 - 3 } \cline { 5 - 6 } Parameter & $f(T)_{P L}$ & $f(T)_{E X P}$ & & $f(T)_{P L}$ & $f(T)_{E X P}$ \\
\hline$\Omega_{b} h^{2}$ & $0.0221_{-0.0003}^{+0.0003}$ & $0.0224_{-0.0002}^{+0.0002}$ & & $0.0222_{-0.0003}^{+0.0003}$ & $0.0224_{-0.0002}^{+0.0002}$ \\
$\Omega_{c} h^{2}$ & $0.1202_{-0.0021}^{+0.0026}$ & $0.1173_{-0.0015}^{+0.0015}$ & $0.1199_{-0.0021}^{+0.0021}$ & $0.1172_{-0.0006}^{+0.0005}$ \\
$n$ & $-0.1967_{-0.1782}^{+0.191}$ & - & $-0.1880_{-0.1171}^{+0.0982}$ & - \\
$p$ & - & $>4.0815$ & - & $>4.0483$ \\
$\Omega_{m}$ & $0.2871_{-0.0095}^{+0.0096}$ & $0.2949_{-0.0084}^{+0.0088}$ & $0.2866_{-0.0022}^{+0.0017}$ & $0.2941_{-0.0019}^{+0.0020}$ \\
$H_{0}$ & $70.40_{-1.14}^{+1.25}$ & $68.83_{-0.68}^{+0.69}$ & $70.41_{-0.41}^{+0.51}$ & $68.89_{-0.22}^{+0.21}$ \\
\hline
\end{tabular}

and $\Omega_{m}-p$ planes. The current only and the current+SL 30 -year results are shown in white and blue, respectively. For the current-only data, the precisions of $\Omega_{m}$ and $H_{0}$ are constrained to the $4.13 \%$ and $1.41 \%$ level, respectively, whereas for the current+SL 30-year data, the precisions of $\Omega_{m}$ and $H_{0}$ are constrained to the $0.94 \%$ and $0.44 \%$ level, respectively. The improvements are also remarkable. However, these results show that the parameter $p$ cannot be well constrained with either the current data or the current+SL 30-year data, and that the SL test data cannot affect the fit results of the model parameter $p$.

From the left-hand panels in Figures 1 and 2, we can conclude that for the considered $f(T)$ models, future SL test data can efficiently break the strong degeneracy between $\Omega_{m}$ and $H_{0}$ existing in the current observational data and thus can greatly improve the precisions of these parameters. The results are consistent with those of our previous studies on dark energy models [10-12]. Thus, we can further confirm that the improvement of parameter estimation by SL test data should be independent of the cosmological models in the background. It is very significant and necessary to include SL test data in future cosmological constraints.

\section{SUMMARY}

The SL test directly measures the temporal variation of the redshift of QSO Lyman- $\alpha$ absorption lines in the so-called "redshift desert" $(2 \lesssim z \lesssim 5)$, which is not covered by any other cosmological observation. In our previous works [10-12], we performed a serious synthetic exploration of the impact of future SL test data on dark energy constraints. It was shown that the SL test can break the parameter degeneracies in existing dark energy probes and significantly improve the precision of dark energy constraints. In particular, combination with the SL test can constrain $\Omega_{m}$ and $H_{0}$ to a high precision for all the considered dark energy models.

In this paper, we quantified the impact of future SL test data on one popular type of MG theory, the $f(T)$ gravity theories. Taking the power-law model $f(T)_{P L}$ and the exponential-form model $f(T)_{E X P}$ as examples, we found that by using a 30-year observation of the SL test, the strong parameter degeneracies of $\Omega_{m}$ and $H_{0}$ can be effectively broken. For the $f(T)_{P L}$ model, the precisions of $\Omega_{m}$ and $H_{0}$ based only on current data are constrained to the $4.70 \%$ and $2.40 \%$ level, whereas those based on current+SL 30-year data are constrained to the $0.97 \%$ and $0.93 \%$ level, respectively. For the $f(T)_{E X P}$ model, the precisions of $\Omega_{m}$ and $H_{0}$ based only on current data are constrained to the $4.13 \%$ and $1.41 \%$ level, whereas those based on current+SL 30-year data are constrained to the $0.94 \%$ and $0.44 \%$ level, respectively. Thus, the constraint precisions of $\Omega_{m}$ and $H_{0}$ can be improved enormously for the considered $f(T)$ models. However, a 30-year observation of the SL test can improve the precision of the model parameter $n$ for the $f(T)_{P L}$ model only moderately and evidently cannot affect the precision of the model parameter $p$ for the $f(T)_{E X P}$ model.

The results for $f(T)$ theories are consistent with those for the dark energy models. We conclude that the improvement of the constraint precision by SL test data is independent of the cosmological models in the background. To make this conclusion more convincing, more MG models other than the $f(T)$ models should be explored, such as the Dvali-Gabadadze-Porrati model [41] and different $f(R)$ models $[44,45]$. We leave a complete analysis of MG theories as future work.

\section{Acknowledgments}

This work was supported by the National Natural Science Foundation of China under Grants No. 11175042 and No. 11522540, the Provincial Department of Education of Liaoning under Grant No. L2012087, and the Fundamental Research Funds for the Central Universities under Grants No. N140505002, No. N140506002, and No. N140504007. 
[1] A. Sandage, The Change of Redshift and Apparent Luminosity of Galaxies due to the Deceleration of Selected Expanding Universes, Astrophys. J. 136, 319 (1962).

[2] A. Loeb, Direct Measurement of Cosmological Parameters from the Cosmic Deceleration of Extragalactic Objects, Astrophys. J. 499, L111 (1998)

[3] P. S. Corasaniti, D. Huterer and A. Melchiorri, Exploring the Dark Energy Redshift Desert with the Sandage-Loeb Test, Phys. Rev. D 75, 062001 (2007)

[4] A. Balbi and C. Quercellini, The time evolution of cosmological redshift as a test of dark energy, Mon. Not. Roy. Astron. Soc 382, 1623 (2007)

[5] H. B. Zhang, W. Zhong, Z. H. Zhu and S. He, Exploring holographic dark energy model with Sandage-Leob test, Phys. Rev. D 76, 123508 (2007)

[6] J. Zhang, L. Zhang and X. Zhang, Sandage-Loeb test for the new agegraphic and Ricci dark energy models, Phys. Lett. B 691, 11 (2010)

[7] Z. Li, K. Liao, P. Wu, H. Yu and Z. H. Zhu, Probing modified gravity theories with the Sandage-Loeb test, Phys. Rev. D 88, 2, 023003 (2013)

[8] S. Yuan, S. Liu and T. J. Zhang, Breaking through the high redshift bottleneck of Observational Hubble parameter Data: The Sandage-Loeb signal Scheme, J. Cosmol. Astropart. Phys. 02, 025 (2015)

[9] M. Martinelli, S. Pandolfi, C. J. A. P. Martins and P. E. Vielzeuf, Probing dark energy with redshift-drift, Phys. Rev. D 86, 123001 (2012)

[10] J. J. Geng, J. F. Zhang and X. Zhang, Quantifying the impact of future Sandage-Loeb test data on dark energy constraints, J. Cosmol. Astropart. Phys. 07, 006 (2014)

[11] J. J. Geng, J. F. Zhang and X. Zhang, Parameter estimation with Sandage-Loeb test, J. Cosmol. Astropart. Phys. 12, 018 (2014)

[12] J. J. Geng, Y. H. Li, J. F. Zhang and X. Zhang, Redshift drift exploration for interacting dark energy, Eur. Phys. J. $C 75$, no. 8, 356 (2015)

[13] J. Liske, A. Grazian, E. Vanzella, M. Dessauges, M. Viel, L. Pasquini, M. Haehnelt and S. Cristiani et al., Cosmic dynamics in the era of Extremely Large Telescopes, Mon. Not. Roy. Astron. Soc 386, 1192 (2008)

[14] P. J. E. Peebles and B. Ratra, Cosmology with a Time Variable Cosmological Constant, Astrophys. J. 325, L17 (1988)

[15] R. R. Caldwell, Spintessence! New models for dark matter and dark energy, Phys. Lett. B 545, 23 (2002)

[16] C. Armendariz-Picon, T. Damour and V. Mukhanov, kinflation, Phys. Lett. B 458, 209 (1999)

[17] A. Y. Kamenshchik, U. Moschella and V. Pasquier, An Alternative to quintessence, Phys. Lett. B 511, 265 (2001)

[18] X. Zhang, F. Q. Wu and J. F. Zhang, New generalized Chaplygin gas as a scheme for unification of dark energy and dark matter, J. Cosmol. Astropart. Phys. 01, 003 (2006)

[19] T. Padmanabhan, Accelerated expansion of the universe driven by tachyonic matter, Phys. Rev. D 66, 021301 (2002)

[20] M. Li, A model of holographic dark energy, Phys. Lett. $B$ 603, 1 (2004)

[21] X. Zhang and F. Q. Wu, Constraints on Holographic
Dark Energy from Latest Supernovae, Galaxy Clustering, and Cosmic Microwave Background Anisotropy Observations, Phys. Rev. D 76, 023502 (2007)

[22] X. Zhang, Heal the world: Avoiding the cosmic doomsday in the holographic dark energy model, Phys. Lett. B 683, 81 (2010)

[23] Y. H. Li, S. Wang, X. D. Li and X. Zhang, Holographic dark energy in a Universe with spatial curvature and massive neutrinos: a full Markov Chain Monte Carlo exploration, J. Cosmol. Astropart. Phys. 02, 033 (2013)

[24] H. Wei, R. G. Cai, and D. F. Zeng, Hessence: A new view of quintom dark energy, Class. Quant. Grav. 22, 3189 (2005)

[25] W. Zhao and Y. Zhang, The state equation of the YangMills field dark energy models, Class. Quant. Grav. 23, 3405 (2006)

[26] X. Zhang, Reconstructing holographic quintessence, Phys. Lett. B 648, 1 (2007)

[27] Y. H. Li, J. F. Zhang and X. Zhang, Parametrized Post-Friedmann Framework for Interacting Dark Energy, Phys. Rev. D 90, 063005 (2014)

[28] Y. H. Li, J. F. Zhang and X. Zhang, Exploring the full parameter space for an interacting dark energy model with recent observations including redshift-space distortions: Application of the parametrized post-Friedmann approach, Phys. Rev. D 90, 123007 (2014)

[29] S. Wang, J. J. Geng, Y. L. Hu and X. Zhang, Revisit of constraints on holographic dark energy: SNLS3 dataset with the effects of time-varying $\beta$ and different light-curve fitters, Sci. China Phys. Mech. Astron. 58(1), 019801 (2015)

[30] M. Zhang, C. Y. Sun, Z. Y. Yang and R. H. Yue, Cosmological evolution of quintessence with a sign-changing interaction in dark sector, Sci. China Phys. Mech. Astron. 57(9), 1805-1808 (2014)

[31] Y. Z. Hu, M. Li, X. D. Li and Z. H. Zhang, Investigating the possibility of a turning point in the dark energy equation of state, Sci. China Phys. Mech. Astron. 57(8), 1607-1612 (2014)

[32] J. B. Lu, L. D. Chen, L. X. Xu and T. Q. Li, Comparing the VGCG model as the unification of dark sectors with observations, Sci. China Phys. Mech. Astron. 57(4), 796$800(2014)$

[33] J. F. Zhang, L. A. Zhao and X. Zhang, Revisiting the interacting model of new agegraphic dark energy, Sci. China Phys. Mech. Astron. 57(2), 387-392 (2014)

[34] X. X. Duan, Y. C. Li and C. J. Gao, Constraining the lattice fluid dark energy from SNe Ia, BAO and OHD, Sci. China Phys. Mech. Astron. 56(6), 1220-1226 (2013)

[35] S. Wang, Y. Z. Wang, J. J. Geng and X. Zhang, Effects of time-varying $\beta$ in SNLS3 on constraining interacting dark energy models, Eur. Phys. J. C 74(11), 3148 (2014)

[36] J. F. Zhang, M. M. Zhao, Y. H. Li and X. Zhang, Neutrinos in the holographic dark energy model: constraints from latest measurements of expansion history and growth of structure, J. Cosmol. Astropart. Phys. 04, 038 (2015)

[37] J. F. Zhang, M. M. Zhao, J. L. Cui and X. Zhang, Revisiting the holographic dark energy in a non-flat universe: alternative model and cosmological parameter constraints, Eur. Phys. J. C 74(11), 3178 (2014) 
[38] M. Li, X. D. Li, S. Wang and Y. Wang, Dark energy: A brief review, Front. Phys. 8(6), 828 (2013)

[39] V. Sahni and S. Habib, Does inflationary particle production suggest Omega $(\mathrm{m})$ less than 1? Phys. Rev. Lett. 81,1766 (1998)

[40] L. Parker and A. Raval, Nonperturbative effects of vacuum energy on the recent expansion of the universe, Phys. Rev. D 60, 063512 (1999)

[41] G. Dvali, G. Gabadadze and M. Porrati, 4-D gravity on a brane in 5-D Minkowski space, Phys. Lett. B 485, 208 (2000)

[42] S. Nojiri, S. D. Odintsov and M. Sasaki, Gauss-Bonnet dark energy, Phys. Rev. D 71, 123509 (2005)

[43] A. Nicolis, R. Rattazzi and E. Trincherini, The Galileon as a local modification of gravity, Phys. Rev. D 79, 064036 (2009)

[44] W. Hu and I. Sawicki, Models of $\mathrm{f}(\mathrm{R})$ Cosmic Acceleration that Evade Solar-System Tests, Phys. Rev. D 76, 064004 (2007)

[45] A. A. Starobinsky, Disappearing cosmological constant in $\mathrm{f}(\mathrm{R})$ gravity, J. Exp. Theor. Phys. Lett. 86, 157 (2007)

[46] G. R. Bengochea and R. Ferraro, Dark torsion as the cosmic speed-up, Phys. Rev. D 79, 124019 (2009)

[47] E. V. Linder, Einstein's Other Gravity and the Acceleration of the Universe, Phys. Rev. D 81, 127301 (2010)

[48] T. Harko, F. S. N. Lobo, S. Nojiri and S. D. Odintsov, f(R,T) gravity, Phys. Rev. D 84, 024020 (2011)

[49] P. Wu and H. W. Yu, The dynamical behavior of $f(T)$ theory, Phys. Lett. B 692, 176 (2010)

[50] R. Zheng and Q. G. Huang, Growth factor in $f(T)$ gravity, J. Cosmol. Astropart. Phys. 03, 002 (2011)

[51] W. Tower, Modified entropic gravity revisited, Sci. China Phys. Mech. Astron. 57(9), 1623-1629 (2014)

[52] J. Wu, Z. X. Li, P. X. Wu and H. W. Yu, Constrains on $\mathrm{f}(\mathrm{T})$ gravity with the strong gravitational lensing data, Sci. China Phys. Mech. Astron. 57(5), 988-993 (2014)

[53] Y. K. Tang, H. S. Zhang, C. Y. Chen and X. Z. Li, Fluctuation with dust of de Sitter ground state of scalar-tensor gravity, Sci. China Phys. Mech. Astron. 57(3), 411-417 (2014)

[54] S. Wang, Y. Z. Wang and X. Zhang, Effects of a TimeVarying Color-Luminosity Parameter $\beta$ on the Cosmological Constraints of Modified Gravity Models, Commun. Theor. Phys. 62(6), 927 (2014)
[55] J. F. Zhang, Y. H. Li and X. Zhang, Measuring growth index in a universe with sterile neutrinos, Phys. Lett. $B$ 739, $102(2014)$

[56] Y. H. Li, J. F. Zhang and X. Zhang, Probing $f(R)$ cosmology with sterile neutrinos via measurements of scaledependent growth rate of structure, Phys. Lett. B 744, 213 (2015)

[57] A. Conley et al., Supernova Constraints and Systematic Uncertainties from the First 3 Years of the Supernova Legacy Survey, Astrophys. J. Suppl. 192, 1 (2011)

[58] G. Hinshaw et al., Nine-Year Wilkinson Microwave Anisotropy Probe (WMAP) Observations: Cosmological Parameter Results, Astrophys. J. Suppl. 208, 19 (2013)

[59] F. Beutler, C. Blake, M. Colless, D. H. Jones, L. StaveleySmith, L. Campbell, Q. Parker and W. Saunders et al., The 6dF Galaxy Survey: Baryon Acoustic Oscillations and the Local Hubble Constant, Mon. Not. Roy. Astron. Soc. 416, 3017 (2011)

[60] N. Padmanabhan, X. Xu, D. J. Eisenstein, R. Scalzo, A. J. Cuesta, K. T. Mehta and E. Kazin, A 2 per cent distance to $z=0.35$ by reconstructing baryon acoustic oscillations - I. Methods and application to the Sloan Digital Sky Survey, Mon. Not. Roy. Astron. Soc. 427, no. 3, $2132(2012)$

[61] L. Anderson, E. Aubourg, S. Bailey, D. Bizyaev, M. Blanton, A. S. Bolton, J. Brinkmann and J. R. Brownstein et al., The clustering of galaxies in the SDSS-III Baryon Oscillation Spectroscopic Survey: Baryon Acoustic Oscillations in the Data Release 9 Spectroscopic Galaxy Sample, Mon. Not. Roy. Astron. Soc. 427, no. 4, 3435 (2013)

[62] C. Blake, S. Brough, M. Colless, C. Contreras, W. Couch, S. Croom, D. Croton and T. Davis et al., The WiggleZ Dark Energy Survey: Joint measurements of the expansion and growth history at $z<1$, Mon. Not. Roy. Astron. Soc. 425, 405 (2012)

[63] Y. Wang and S. Wang, Distance Priors from Planck and Dark Energy Constraints from Current Data, Phys. Rev. D 88, 043522 (2013)

[64] A. G. Riess et al., A 3\% Solution: Determination of the Hubble Constant with the Hubble Space Telescope and Wide Field Camera 3, Astrophys. J. 730, 119 (2011) 